ASTHMA

\title{
Pathogenic bacteria and viruses in induced sputum or pharyngeal secretions of adults with stable asthma
}

\author{
T H Harju, M Leinonen, J Nokso-Koivisto, T Korhonen, R Räty, Q He, T Hovi, \\ J Mertsola, A Bloigu, P Rytilä, P Saikku
}

See end of article for authors' affiliations

.....................

Correspondence to: Dr T H Hariu, Department of Internal Medicine, P O Box 5000, 90014 University of Oulu, Finland; terttu.harju@ oulu.fi

Received

25 November 2005

Accepted

28 February 2006

Published Online First

3 March 2006

\begin{abstract}
Background: Respiratory infections are well known triggers of asthma exacerbations, but their role in stable adult asthma remains unclear.

Methods: 103 asthmatics and 30 control subjects were enrolled in the study. Sputum was induced by inhalation of $3 \% \mathrm{NaCl}$ solution. Oropharyngeal swab specimens were obtained from the posterior wall of the oropharynx. Respiratory specimens were analysed by RT-PCR for rhinovirus, enterovirus and respiratory syncytial virus and by PCR for adenovirus, Chlamydia pneumoniae, Mycoplasma pneumoniae and Bordetella pertussis.

Results: Sputum samples from two of the 30 healthy controls (6.7\%), five of 53 patients with mild asthma $(9.4 \%)$, and eight of 50 with moderate asthma (16.0\%) were positive for rhinovirus. Rhinovirus positive asthmatic subjects had more asthma symptoms and lower forced expiratory volume in 1 second $\left(F V_{1}\right)$ (79\% predicted) than rhinovirus negative cases (93.5\% predicted; $p=0.020)$. Chlamydia pneumoniae PCR was positive in 11 healthy controls (36.6\%), 11 mild asthmatics (20.8\%), and 11 moderate asthmatics $(22 \%)$, and PCR positive asthmatics had lower $\mathrm{FEV}_{1} / \mathrm{FVC}$ than negative cases $(78.2 \% v 80.8 \%, \mathrm{p}=0.023)$. Bordetella pertussis PCR was positive in 30 cases: five healthy controls (16.7\%), 15 mild asthmatics (28.3\%), and 10 moderate asthmatics (20\%). Bordetella pertussis positive individuals had lower $\mathrm{FEV}_{1} / \mathrm{FVC}$ $(77.1 \%$ v $80.7 \%, p=0.012)$ and more asthma symptoms than $B$ pertussis negative cases.

Conclusions: Rhinovirus, $C$ pneumoniae, and $B$ pertussis are found in the sputum or pharyngeal swab specimens of asthmatic subjects without concurrent symptoms of infection or asthma exacerbation, as well as in some healthy controls. Positivity is associated with lower lung function and more frequent asthma symptoms.
\end{abstract}

$\mathrm{T}$ he aetiology of asthma involves interactions between genetic susceptibility, allergen exposure, and external aggravating factors such as air pollution, smoking and respiratory infections. Both clinical and experimental evidence suggest an important role for respiratory infections as triggers of asthma attacks in adults and in children. Viral respiratory infections are considered the most common precipitating factors of acute asthma and have been shown to be associated with over $80 \%$ of asthma exacerbations in school children. ${ }^{1}$ In that study picornaviruses (mostly rhinoviruses) accounted for two thirds of the viral infections, with coronavirus causing less severe asthma exacerbations than other respiratory viruses. Thumerelle et $a^{2}$ confirmed the high incidence of viral infections (especially enteroviruses or rhinoviruses) in children admitted to hospital with an asthma exacerbation. Persistent clinical symptoms were more commonly associated with atypical bacterial infections.

Atypical bacteria, Chlamydia pneumoniae, and Mycoplasma pneumoniae infections may precede asthma onset or exacerbate asthma, ${ }^{3}$ and these bacteria might be involved in chronic asthma. In vitro and animal studies suggest that atypical agents play a role in the pathogenesis of the disease. ${ }^{4}$ The association between $C$ pneumoniae infection and adult onset asthma was first described by Hahn et al in 1991. ${ }^{5}$ There is also evidence that $C$ pneumoniae may be involved in the pathogenesis of chronic stable asthma: serological studies suggest a dose response between $C$ pneumoniae antibody levels and the severity of asthma ${ }^{6}$ and reactivation of $C$ pneumoniae infection during acute asthma. ${ }^{7}$ Further evidence of a possible role of $C$ pneumoniae in asthma is derived from observations that some subjects treated with antichlamydial antibiotics reported improvement in their asthma symptoms. ${ }^{8}$ The role of $M$ pneumoniae in stable chronic asthma has been studied recently by Kraft et al and treatment with claritromycin led to a significant improvement in lung function in the polymerase chain reaction (PCR) positive subjects.

Bordetella pertussis, the causative agent of whooping cough, produces toxins which cause damage to the epithelium and depress the defence mechanisms. The role of $B$ pertussis in asthma is not known, but it has been shown that asthmatics with whooping cough suffer longer from cough and have an increased risk for sinusitis. ${ }^{10}$

The potential role of asymptomatic viral infections in asthma is poorly defined. In a study of asymptomatic asthmatic children over $80 \%$ virus positivity has been reported by PCR, while 5\% of swabs from healthy controls were virus positive. ${ }^{11}$ In children with acute expiratory wheezing, viral RNA may take up to 5 weeks after the infection to disappear from the nasal mucus. ${ }^{12}$

We hypothesised that respiratory viruses as well as $C$ pneumoniae, $M$ pneumoniae, and B pertussis are present in the airways of asthmatics, and that these infectious agents may be found in induced sputum and/or pharyngeal secretions of

Abbreviations: $\mathrm{CRP}, \mathrm{C}$-reactive protein; $\mathrm{FEV}_{1}$, forced expiratory volume in 1 second; FVC, forced vital capacity; PCR, polymerase chain reaction; RSV, respiratory syncytial virus 
Table 1 Demographic features and lung function of study groups

\begin{tabular}{|c|c|c|c|c|}
\hline & $\begin{array}{l}\text { Healthy controls } \\
(\mathrm{N}=30)\end{array}$ & $\begin{array}{l}\text { Mild asthma } \\
(\mathrm{N}=53)\end{array}$ & $\begin{array}{l}\text { Moderate asthma } \\
(\mathrm{N}=50)\end{array}$ & $p$ value \\
\hline Mean (SD) age (years) & $39(14)$ & $43(13)$ & 45 (12) & $0.075^{\star}$ \\
\hline $\operatorname{Sex}(M: F)$ & $10: 20$ & $14: 39$ & $17: 33$ & $0.668 \ddagger$ \\
\hline Smoking (non:current) & $27: 3$ & $48: 5$ & $38: 12$ & $0.097 \S$ \\
\hline Atopy $\%$ (n)†† & $25 \%(8)$ & $42 \%(22)$ & $54 \%(27)$ & $0.034 \ddagger$ \\
\hline $\begin{array}{l}\text { Median (IQR) inhaled } \\
\text { corticosteroids ( } \mu \mathrm{g} / \text { day) }\end{array}$ & & $500(400-800)$ & $900(475-1600)$ & $0.006^{* *}$ \\
\hline Median (IQR) duration of asthma & & $1(0.5-1.5)$ & $3(0.5-9)$ & $0.010^{* *}$ \\
\hline (years), range & & $0.1-39$ & $0.1-31$ & \\
\hline Mean (SD) FEV & $3.56(0.78)$ & $3.41(0.82)$ & $2.7(0.61) \dagger$ & $<0.001^{*}$ \\
\hline Mean (SD) FEV $1 \%$ predicted) & $97.6(11)$ & $101(11)$ & $80.5(13) \dagger$ & $<0.001^{*}$ \\
\hline Mean (SD) FVC & $4.33(0.98)$ & $4.2(1.03)$ & $3.57(0.82) \dagger$ & $0.001^{*}$ \\
\hline Mean (SD) FVC (\% predicted) & $99.0(11)$ & $103(12)$ & $87.1(12) \dagger$ & $<0.001^{*}$ \\
\hline Mean (SD) $\mathrm{FEV}_{1} / \mathrm{FVC} \%$ & $82.6(5.9)$ & $81.6(5.1)$ & $76.4(8.1) \dagger$ & $<0.001^{*}$ \\
\hline Mean (SD) $\mathrm{FEV}_{1} / \mathrm{FVC}$ (\% predicted) & $98.1(7.2)$ & $97.9(7.4)$ & $92(8.9) \dagger$ & $<0.001^{*}$ \\
\hline \multicolumn{5}{|c|}{$\begin{array}{l}\text { *Analysis of variance. } \\
\dagger \text { The mean difference is significant at the } 0.05 \text { level } v \text { healthy controls (Dunnett } t \text { test). } \\
\ddagger \chi^{2} \text { test. } \\
\$ \text { Fisher's exact test. } \\
\text { Tp } 0.010 \text { between moderate asthma and healthy controls ( } \chi^{2} \text { test). } \\
\text { **Mann-Whitney } U \text { test between mild and moderate asthma groups. } \\
\dagger † \text { Anamnestic information of prick test positivity. }\end{array}$} \\
\hline
\end{tabular}

asthmatics. Sensitive assay for C-reactive protein was used as a marker of systemic inflammation.

\section{METHODS}

\section{Study population}

One hundred and three asthmatics with disease severity ranging from mild $(n=53)$ to moderate $(n=50)$ and 30 control subjects were included in the study during 1999. Their demographic characteristics and lung function are shown in table 1 .

The clinical severity of asthma was classified according to the GINA guidelines. ${ }^{13}$ Patients with asthma were recruited from the outpatient clinic of Oulu University Hospital and the Department of Allergy, Helsinki University Central Hospital. Asthma patients fulfilling the study criteria were asked to participate in the study when they came to the lung function laboratory to perform spirometric tests between 7 December 1998 and 16 December 1999, excluding the summer months. Induced sputum and oropharyngeal specimens were obtained from individuals at the same session/visit. Healthy controls were volunteers with no lung disease and normal lung function. The mild asthma group included 33 asthmatics fulfilling the American Thoracic Society asthma criteria and 20 subjects with mild intermittent asthma (asthma symptoms, bronchial hyperreactivity, exclusion of other lung diseases but less than $15 \%$ forced expiratory volume in 1 second $\left(\mathrm{FEV}_{1}\right)$ reversibility). Both controls and asthmatic patients had been free from respiratory infection for at least 4 weeks. Asthma symptoms during the last month (cough, sputum production, shortness of breath, wheezing or cough at exercise, and disturbed sleep) were recorded on a structured questionnaire and graded on a scale ranging from 0 (asymptomatic) to 3 (the most severe discomfort), and the frequency of asthma symptoms was graded from 0 (no asthma symptoms) to 3 (several times per day). The results were combined to form an asthma severity score.

The study protocol was approved by the ethics committees of the University of Oulu and Oulu University Hospital and of Helsinki University Central Hospital. All subjects gave their informed consent.

\section{Sputum induction}

Sputum was induced by inhalation of $5 \mathrm{ml} 3 \% \mathrm{NaCl}$ solution using an ultrasonic nebuliser (Omron U1, Omron, Germany). Both healthy controls and asthmatics were given salbutamol
$0.2 \mathrm{mg}$ as a dry powder inhalation 15 minutes before sputum induction (Buventol Easyhaler $0.1 \mathrm{mg} /$ dose, Orion Pharma, Espoo, Finland). The sputum was processed as previously described. ${ }^{14}$ The filtered suspension was centrifuged and the cell pellet and supernatant were stored at $-70^{\circ} \mathrm{C}$ for later assays. In 67 cases ( 17 controls, 26 patients with mild asthma and 24 with moderate asthma) cytospin slides were made for the assessment of the cell differential count.

\section{Pharyngeal swab}

Oropharyngeal swab specimens were obtained from the posterior wall of the oropharynx with a sterile cotton tipped swab and placed in Chlamydia Transit medium and frozen at $-70^{\circ} \mathrm{C}$.

\section{Measurement of CRP concentration}

Serum CRP levels were measured with a rapid two-site ultrasensitive assay based on time-resolved immunofluorometry. ${ }^{15}$

\section{RT-PCR hybridisation method for rhinovirus and entrovirus}

RNA was extracted from $100 \mu$ of clinical specimen using a commercial kit (RNeasy, Qiagen, GmbH, Heidelberg, Germany) and the specimens were analysed for rhinovirus and enterovirus RNA by a modification of the previously described RT-PCR hybridisation method ${ }^{16}$ using two different lanthanide labelled probes designed for detection of amplicons representing enteroviruses or rhinoviruses, respectively. ${ }^{17}$ A positive result in two parallel wells was required to classify a specimen as positive. If only one well was positive, the specimen was re-assayed. The specimen was regarded rhinovirus positive if the rhinovirus probe yielded a positive result, and enterovirus positive if the enterovirus probe alone was positive. ${ }^{16}{ }^{17}$

\section{RT-PCR for respiratory syncytial virus (RSV)}

RSV detection by RT-PCR was a modification of the methods described before. Briefly, purified RNA was reverse transcribed and the resulting cDNA was amplified using the oligonucleotide primers described by Osiowy. ${ }^{18}$ The 5 end of the reverse primer was labelled with biotin. The RSV type was identified by a liquid phase hybridisation method ${ }^{16}$ using RSV A and RSV B specific lanthanide labelled probes. 


\begin{tabular}{|c|c|c|c|c|}
\hline & $\begin{array}{l}\text { Healthy controls } \\
(\mathrm{N}=30)\end{array}$ & $\begin{array}{l}\text { Mild asthma } \\
(\mathrm{N}=53)\end{array}$ & $\begin{array}{l}\text { Moderate asthma } \\
(\mathrm{N}=50)\end{array}$ & p value ${ }^{*}$ \\
\hline Adenovirus & 0 & 0 & 0 & \\
\hline Enterovirus & $2(6.7 \%)$ & 0 & $1(2 \%)$ & 0.118 \\
\hline RSV & 0 & 0 & 0 & \\
\hline Rhinovirus & $2(6.7 \%)$ & $5(9.4 \%)$ & $8(16.0 \%)$ & 0.438 \\
\hline Chlamydia pneumoniae & $9(30 \%)$ & $10(18.9 \%)$ & $8(16 \%)$ & 0.304 \\
\hline Mycoplasma pneumoniae & $1(3.3 \%)$ & 0 & 0 & 0.226 \\
\hline Bordetella pertussis & $3(10 \%)$ & $7(13 \%)$ & $3(6 \%)$ & 0.561 \\
\hline
\end{tabular}

\section{PCR for Chlamydia pneumoniae}

DNA from nasopharyngeal swab and sputum specimens was isolated using Qiamp DNA Mini kits (QIAGEN). Quantitative LightCycler real time PCR assay was used to detect the presence of $C$ pneumoniae 16S rDNA in the samples. ${ }^{19}$ As a standard, a dilution series of 80000 to 8 genome equivalents of $C$ pneumoniae DNA extracted from $C$ pneumoniae elementary bodies (strain Kajaani 7) was used. The sample volume was $8 \mu \mathrm{l}$ and a negative control was included as every seventh sample.

\section{PCR for Mycoplasma pneumoniae and adenovirus}

The PCR methods for detection of adenoviruses and $M$ pneumoniae were performed as described elsewhere. ${ }^{2021}$

\section{PCR for Bordetella pertussis}

Details of the PCR have been described earlier and the assay has proved to be specific for $B$ pertussis. ${ }^{22}$ Primers targeting insertion sequence 481 of $B$ pertussis were used and 40 cycles were carried out. The DNA extracted from the prototype strain of $B$ pertussis was used as the positive control, and all reagents except template DNA were included in the negative control tube. Both positive and negative controls were included in each PCR run. The amplified products were separated by $1.5 \%$ agarose gel electrophoresis and the bands were visualised after staining with ethidium bromide. The sensitivity of the PCR assay was $\sim 5$ bacteria per reaction tube. ${ }^{22}$

\section{Statistical methods}

The results are expressed as mean (SD) or as median (range) or (interquartile range, IQR) for continuous variables and as frequencies or percentages for categorical variables. Analysis of variance followed by the Dunnett post-test was used to compare continuous variables between the three study groups. In the case of categorical variables, the groups were compared using a $\chi^{2}$ test or Fisher's exact test, as appropriate. The variables representing the use of inhaled corticosteroids and the duration of asthma were skewed and applicable only for two asthma groups; comparisons between the groups were therefore performed using the non-parametric
Mann-Whitney U test. The Mann-Whitney U test was used for comparisons of lung function and CRP between negative and positive cases because of the small data sets. The CIA program was used to calculate $95 \%$ confidence intervals for median differences.

\section{RESULTS}

The microbiological findings in induced sputum of asthma patients and controls are shown in table 2. Four asthmatics were positive for both rhinovirus and $C$ pneumoniae, and three healthy controls and seven asthmatics were positive for both $B$ pertussis and C pneumoniae. Adenovirus and RSV were not found in the sputum of patients or controls. Two healthy controls and one asthma patient had enterovirus in their sputum. One healthy control had M pneumoniae DNA in the cellular fraction of her sputum. All other cases were mycoplasma negative.

Sputum cell differential counts were available for 17 controls, 26 cases with mild asthma, and 24 cases with moderate asthma. The mean (SD) percentage of eosinophils was higher in asthmatics than in healthy controls $(0.24$ $(0.73) \%$ in controls, $6.06(15.42) \%$ in patients with mild asthma, and $4.36(7.14) \%$ in patients with moderate asthma; $\mathrm{p}=0.005$ ). In individuals positive for $B$ pertussis PCR there was mild lymphocytosis in the induced sputum differential cell count $(0.4 \%$ in B pertussis negative cases $v 5.2 \%$ in positive cases, $p=0.039$ ). There was no correlation between the differential cell count and positivity for other studied infectious agents.

Rhinoviruses were found by PCR in 15 sputum samples (nine cellular fractions, four supernatants, and two both fractions, table 3 ) in two of 30 healthy controls $(6.7 \%)$, five of 53 mild asthmatics $(9.4 \%)$, and eight of 50 patients with moderate asthma (16.0\%). Rhinovirus positive cases more often had moderate asthma, but this trend was not statistically significant $(p=0.179)$. Rhinovirus positive asthma patients had lower $\mathrm{FEV}_{1}$ than rhinovirus negative patients (median 79\% predicted $v$ 93.5\% predicted, $\mathrm{p}=0.020$ ), whereas in healthy controls no differences were seen between rhinovirus positive and negative individuals. Rhinovirus PCR positive cases also had higher asthma

Table 3 Microbiological findings (rhinovirus, Bordetella pertussis and Chlamydia pneumoniae) in different fractions of induced sputum and nasopharyngeal swabs

\begin{tabular}{llll}
\hline & $\begin{array}{l}\text { Rhinovirus } \\
(\mathrm{N}=133)\end{array}$ & $\begin{array}{l}\text { Bordetella pertussis } \\
(\mathrm{N}=127)\end{array}$ & $\begin{array}{l}\text { Chlamydia } \\
\text { pneumoniae } \\
(\mathrm{N}=130)\end{array}$ \\
\hline $\begin{array}{l}\text { Pharyngeal swab } \\
\text { Induced sputum, supernatant }\end{array}$ & $\mathrm{NT}$ & $22(17.3 \%)^{*}$ & $6(4.9 \%)$ \\
Induced sputum, cellular fraction & $11(4.5 \%)$ & $8(6.3 \%)^{*}$ & $14(11.1 \%)$ \\
\hline
\end{tabular}

NT, not tested.

*Positivity rate for $B$ pertussis in pharyngeal swab and cellular fraction ( $p=0.001$, McNemar test), supernatant or pharyngeal swab (0=0.017, McNemar test). 


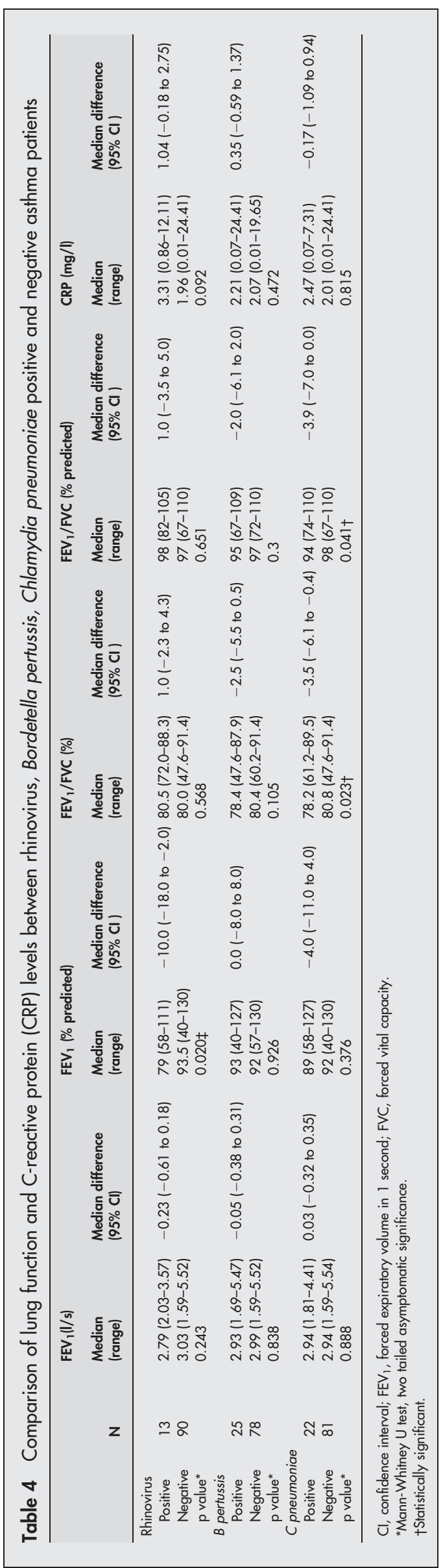

symptom scores (above the median in $80 \%$ of rhinovirus positive cases compared with $47.4 \%$ of rhinovirus negative cases, $p=0.018)$. There was a trend for higher CRP levels in rhinovirus positive subjects (median $3.31 \mathrm{mg} / \mathrm{l} v 1.96 \mathrm{mg} / \mathrm{l}$ ), but the difference was not statistically significant $(\mathrm{p}=0.092)$. $40 \%$ of rhinopositive cases were atopic compared with $43 \%$ of rhinovirus negative cases $(\mathrm{p}=0.812)$.

Chlamydia pneumoniae PCR was positive in 33 cases: 11 healthy controls (36.7\%), 11 mild asthmatics (20.8\%), and 11 moderate asthmatics $(22 \%, \mathrm{p}=0.230)$. The positive sample was most often induced sputum (31 positive findings: 17 cellular fraction, 14 supernatant, and in four cases both fractions were positive, table 3). Pharyngeal swabs were positive in only six cases. Chlamydia pneumoniae positive asthma patients had more airways obstruction (median $\mathrm{FEV}_{1} / \mathrm{FEV} 78.3 \% \quad v \quad 80.8 \%, \mathrm{p}=0.023$ and $94 \% \quad v 98 \%$ of predicted, $\mathrm{p}=0.041)$ and the duration of asthma tended to be shorter (median $0.5 v 1$ years, $\mathrm{p}=0.089$ ). Interestingly, CRP levels in $C$ pneumoniae positive healthy controls, but not in asthma patients, were significantly lower than in negative individuals (median $0.42 v 1.28, \mathrm{p}=0.004$ ).

Bordetella pertussis PCR was positive in 30 cases: five healthy controls (16.7\%), 15 mild asthmatics $(28.3 \%)$, and 10 moderate asthmatics (20.0\%, $\mathrm{p}=0.894$; table 2 ). Pharyngeal swabs were most often the positive sample (25 positive findings $v$ five sputum/cellular fraction and eight sputum/ supernatant). In one case all samples were positive. In healthy controls $3 / 5$ positive findings were in pharyngeal swabs compared with $12 / 15$ patients with mild asthma and 7/ 10 with moderate asthma. Bordetella pertussis positive healthy controls had lower $\mathrm{FEV}_{1} / \mathrm{FVC}$ (median $92.0 \%$ v $98 \%$ predicted, $\mathrm{p}=0.019$, table 4 ).

The asthma symptom score was higher in $B$ pertussis positive cases than in negative cases $(66 \% v 47 \%$ had symptom scores above median, $p=0.053)$. CRP levels did not differ between negative and positive groups (median $1.69 \mathrm{mg} / \mathrm{l} v 1.62 \mathrm{mg} / \mathrm{l}, \mathrm{p}=0.588) .37 \%$ of $B$ pertussis positive cases were atopic compared with $45 \%$ of negative cases $(\mathrm{p}=0.436)$.

\section{DISCUSSION}

Our study has shown that there is an association between pathogen positivity and clinical symptoms in patients with asthma: the presence of genetic material of the studied pathogens was associated with lower lung function and more frequent asthma symptoms. Rhinovirus RNA as well as $C$ pneumoniae and $B$ pertussis DNA are commonly present in the sputum or pharyngeal swab specimens of asthmatic patients without ongoing infection or asthma exacerbation, as well as in healthy controls. Adenovirus and RSV were not found in any of the induced sputum samples by the PCR methods used, and enterovirus and $M$ pneumoniae were present only occasionally.

Several respiratory viruses are known triggers of asthma episodes, and $70-85 \%$ of asthma exacerbations are associated with upper respiratory tract infections, of which $60 \%$ are caused by rhinoviruses. ${ }^{1}$ There are only a few studies in which rhinoviruses have been sought from respiratory tract specimens obtained from healthy persons or from patients with stable asthma. Corne $e a^{23}$ followed 76 couples in which one person had atopic asthma and one was healthy by taking nasal aspirates every second week; rhinoviruses were found in $10.1 \%$ and $8.5 \%$ of specimens obtained from asthmatic and healthy subjects, respectively. In our study, asthmatic patients tended to have higher rhinovirus positivity than healthy controls. Interestingly, we found no rhinoviruses in induced sputum, which can be considered to represent the lower respiratory tract. Rhinovirus is an important cause of exacerbations of a number of pre-existing airways disorders, ${ }^{24}$ 
and recent studies have shown that it can inhabit the lower respiratory tract even in cases where upper respiratory tract specimens are rhinovirus negative..$^{25} 26$

Human rhinovirus has been implicated as the principal virus associated with asthma episodes in children ${ }^{1}$ and in adults. Respiratory virus infections may also contribute to allergic sensitisation to aeroallergens and to the development of asthma. Experimental rhinovirus 16 infection has been found to cause airway obstruction in subjects with atopic asthma, ${ }^{27}$ and to increase intercellular adhesion molecule 1 expression in the bronchial epithelium of asthmatics, regardless of inhaled steroid treatment. Experimental rhinovirus infections have shown a deficient type l cytokine response in asthmatics ${ }^{28}$ and correlation with symptom severity, as well as impaired viral clearance. An impaired innate response to rhinovirus infection in primary bronchial epithelial cell culture from asthmatic subjects has also been found. ${ }^{29}$ Rhinovirus PCR positive individuals may either have a chronic infection or a slowly resolving acute infection. Following PCR positive individuals by sputum sampling at several time points would provide an answer to this question.

In our study we also showed that $C$ pneumoniae DNA can be present in the lower airway secretions of both asthmatics and healthy controls without symptoms of ongoing respiratory infection. Chlamydia pneumoniae seems to be more common in the lower respiratory tract than in the upper respiratory tract, occurring in only $4.9 \%$ of nasopharyngeal specimens and $16 \%$ of induced sputum. Biscione et al ${ }^{30}$ have shown that $C$ pneumoniae is more commonly present in the nasal secretions of asthmatic patients than in non-asthmatic healthy subjects $(6.4 \% \vee 2.3 \%)$. In our study the presence of $C$ pneumoniae in the upper respiratory tract agreed with the finding of Biscione et $a l^{30}$ although we could not find any difference between asthmatics and healthy controls. Wu et al ${ }^{31}$ studied lung tissue samples by immunohistochemical staining for $C$ pneumoniae and found positive staining in $44 \%$ of lung tissues obtained from young persons (mean age 32 years) who had died accidentally, although the number of positive cells was low. In addition, they showed that all lung tissues from elderly persons were $C$ pneumoniae positive, but the number of $C$ pneumoniae positive cells was significantly higher in patients with chronic obstructive pulmonary disease. Thus, C pneumoniae seems to be common in the lower respiratory tract of healthy persons and of those with respiratory diseases. Interestingly, in healthy controls CRP levels were lower in $C$ pneumoniae positive cases than in negative cases, but the opposite was true for asthmatics, which suggests that the presence of $C$ pneumoniae as such does not lead to systemic inflammation. It is possible that genetic and environmental factors determine the outcome and sequelae of persistent $C$ pneumoniae infection in the host.

It is known that in many countries whooping cough is increasingly being recognised as an important cause of respiratory disease in adults. ${ }^{32}$ In Finland, $23-40 \%$ of diagnosed pertussis cases-as defined by culture, PCR and enzyme immunoassay serology-were found in adults in 1995-2004. The present study was carried out in the year 1999 when there was a nationwide epidemic of pertussis in the country. It remains to be shown whether $B$ pertussis DNA found in airway secretions in the study subjects was due to transient colonisation of $B$ pertussis.

Bordetella pertussis respiratory infection has been shown to selectively induce T helper 1 type responses in humans, ${ }^{33}$ and hypothetically to reduce the development of asthma later in life. In a murine model of allergic asthma, Bordetella infection before ovalbumin sensitisation led to increased bronchial hyperreactivity and exacerbation of the allergic asthmatic response but, in already sensitised animals, whole cell $B$ pertussis inhibited the allergic airway reaction induced by ovalbumin..$^{34}$ In our study asthmatics did not have more or less $B$ pertussis positivity in their airway secretions than healthy controls, but those who had $B$ pertussis were more symptomatic and had more airways obstruction. The presence of $B$ pertussis DNA in the airways did not have a protective effect against asthma symptoms or signs. The difference in $\mathrm{FEV}_{1} / \mathrm{FVC}$ between healthy controls who were PCR positive and negative for $B$ pertussis was small; this finding should be interpreted tentatively.

Although adenoviral DNA has been found in bronchial samples from asthmatic and control subjects, ${ }^{35}$ no adenoviral DNA was found in the induced sputum samples of our study material. Cells in induced sputum are mainly alveolar macrophages and polymorphonuclear cells, with only occasional bronchial epithelial cells. It might be possible that, in subjects with stable asthma, adenovirus DNA exists only in bronchial epithelial cells and is not therefore found in induced sputum.

The association between RSV infection and asthma is somewhat controversial, but the available data support the suggestion that RSV infection can facilitate or enhance allergic sensitisation and the development of allergic airway disease, at least in some asthmatic children. ${ }^{36}$ In this study no RSV RNA was found in sputum samples.

Our study showed that rhinovirus as well as $C$ pneumoniae and $B$ pertussis are found in the sputum or pharyngeal swab specimens of asthmatic subjects without concurrent symptoms of infection or asthma exacerbation, as well as in some healthy controls. Positivity is associated with lower lung function only in asthmatics and more frequent asthma symptoms. Patients with asthma have been shown to have an increased risk of invasive pneumococcal disease. ${ }^{37}$ The presence of two pathogens in 14 individuals is an interesting finding, and the significance of the occurrence of several pathogens, host factors leading to different responses to pathogens, and the potential effects of the presence of these organisms on the baseline respiratory function remain to be examined in further studies.

The sample size was small due to the short period of time over which study material was collected. It is possible that some effects have been missed due to low power and the results need to be interpreted with caution.

\section{ACKNOWLEDGEMENTS}

The authors thank Maritta Möller and Tuula Rantasalo for excellent technical assistance (pertussis).

\section{Authors' affiliations}

T H Harju, Department of Internal Medicine, University of Oulu, Oulu, Finland

M Leinonen, J Nokso-Koivisto, T Korhonen, R Räły, Q He, T Hovi, A Bloigu, P Saikku, National Public Health Institute, Oulu, Turku and Helsinki, Finland

J Mertsola, Department of Pediatrics, Turku University Hospital, Turku, Finland

P Rytilä, Department of Allergy, Skin and Allergy Hospital, Helsinki University Central Hospital, Helsinki, Finland

P Saikku, Department of Medical Microbiology, University of Oulu, Oulu, Finland

This work was supported by a grant from the Finnish Anti-Tuberculosis Association Foundation, the Finnish Lung Health Association, and the University of Oulu Scholarship Foundation.

Competing interests: none declared.

\section{REFERENCES}

1 Johnston SL, Pattemore PK, Sanderson G, et al. Community study of role of viral infections in exacerbations of asthma in 9-11 year old children. BMJ 1995;310:1225-9. 
2 Thumerelle C, Deschildre A, Bouquillon C, et al. Role of viruses and atypical bacteria in exacerbations of asthma in hospitalized children: a prospective study in the Nord-Pas de Calais region (France). Pediatr Pulmonol 2003;35:75-82.

3 Daian CM, Wolff AH, Bielory L. The role of atypical organisms in asthma. Allergy Asthma Proc 2000;21:107-11.

4 Blasi F, Cosentini R, Tarsia P, et al. Atypical pathogens and asthma: can they influence the natural history of the disease? Monaldi Arch Chest Dis 2001;56:276-80.

5 Hahn DL, Dodge RW, Golubjatnikov R. Association of Chlamydia pneumoniae (strain TWAR) infection with wheezing, asthmatic bronchitis, and adult-onset asthma. JAMA 1991;266:225-30.

6 Black PN, Scicchitano R, Jenkins CR, et al. Serological evidence of infection with Chlamydia pneumoniae is related to the severity of asthma. Eur Respir J 2000; 15:254-9.

7 Wark PA, Johnston SL, Simpson JL, et al. Chlamydia pneumoniae immunoglobulin A reactivation and airway inflammation in acute asthma. Eur Respir J 2002;20:834-40.

8 Hahn DL. Treatment of Chlamydia pneumoniae infection in adult asthma: a before-after trial. J Fam Pract 1995;41:345-51.

9 Kraft M, Cassell GH, Pak J, et al. Mycoplasma pneumoniae and Chlamydia pneumoniae in asthma: effect of clarithromycin. Chest 2002;121:1782-8.

10 De Serres G, Shadmani R, Duval B, et al. Morbidity of pertussis in adolescents and adults. J Infect Dis 2000;182:174-9.

11 Marin J, Jeler-Kacar D, Levstek V, et al. Persistence of viruses in upper respiratory tract of children with asthma. J Infect 2000;41:69-72.

12 Jartti T, Lehtinen P, Vuorinen T, et al. Persistence of rhinovirus and enterovirus RNA after acute respiratory illness in children. J Med Virol 2004:72:695-9.

13 GINA. Global strategy for asthma management and prevention, NHLBI/NIH Workshop Report 95-3659. Bethesda, MD: National Heart, Lung and Blood Institute, 1995.

14 Rytila P, Metso T, Heikkinen K, et al. Airway inflammation in patients with symptoms suggesting asthma but with normal lung function. Eur Respir $J$ 2000;16:824-30.

15 Jousilahti P, Salomaa V, Hakala K, et al. The association of sensitive systemic inflammation markers with bronchial asthma. Ann Allergy Asthma Immunol 2002;89:381-5.

16 Blomqvist S, Skytta A, Roivainen M, et al. Rapid detection of human rhinoviruses in nasopharyngeal aspirates by a microwell reverse transcriptionPCR-hybridization assay. J Clin Microbiol 1999;37:2813-6.

17 Nokso-Koivisto J, Kinnari TJ, Lindahl P, et al. Human picornavirus and coronavirus RNA in nasopharynx of children without concurrent respiratory symptoms. J Med Virol 2002;66:417-20.

18 Osiowy C. Direct detection of respiratory syncytial virus, parainfluenza virus, and adenovirus in clinical respiratory specimens by a multiplex reverse transcription-PCR assay. J Clin Microbiol 1998;36:3149-54.

19 Reischl U, Lehn N, Simnacher U, et al. Rapid and standardized detection of Chlamydia pneumoniae using LightCycler real-time fluorescence PCR. Eur J Clin Microbiol Infect Dis 2003;22:54-7.
20 Raty R, Kleemola M, Melen K, et al. Efficacy of PCR and other diagnostic methods for the detection of respiratory adenoviral infections. J Med Virol 1999;59:66-72.

21 Raty R, Kleemola M. Detection of Mycoplasma pneumoniae by polymerase chain reaction in middle ear fluids from infants with acute otitis media. Pediatr Infect Dis J 2000;19:666-8.

$22 \mathrm{He} \mathrm{Q}$, Schmidt-Schlapfer G, Just M, et al. Impact of polymerase chain reaction on clinical pertussis research: Finnish and Swiss experiences. J Infect Dis 1996; 174:1288-95.

23 Corne JM, Marshall C, Smith S, et al. Frequency, severity, and duration of rhinovirus infections in asthmatic and non-asthmatic individuals: a longitudinal cohort study. Lancet 2002;359:831-4.

24 Hayden FG. Rhinovirus and the lower respiratory tract. Rev Med Virol 2004; 14:17-31

25 Mosser AG, Vrtis R, Burchell L, et al. Quantitative and qualitative analysis of rhinovirus infection in bronchial tissues. Am J Respir Crit Care Med 2005;171:645-51.

26 Simons E, Schroth MK, Gern JE. Analysis of tracheal secretions for rhinovirus during natural colds. Pediatr Allergy Immunol 2005;16:276-8.

27 Grunberg K, Timmers MC, de Klerk EP, et al. Experimental rhinovirus 16 infection causes variable airway obstruction in subjects with atopic asthma. Am J Respir Crit Care Med 1999;160:1375-80.

28 Papadopoulos NG, Stanciu LA, Papi A, et al. A defective type 1 response to rhinovirus in atopic asthma. Thorax 2002;57:328-32.

29 Wark PA, Johnston SL, Bucchieri F, et al. Asthmatic bronchial epithelial cells have a deficient innate immune response to infection with rhinovirus. J Exp Med 2005;201:937-47.

30 Biscione GL, Corne J, Chauhan AJ, et al. Increased frequency of detection of Chlamydophila pneumoniae in asthma. Eur Respir J 2004;24:745-9.

31 Wu L, Skinner SJ, Lambie N, et al. Immunohistochemical staining for Chlamydia pneumoniae is increased in lung tissue from subjects with chronic obstructive pulmonary disease. Am J Respir Crit Care Med 2000; 162:1148-51.

32 von Konig $\mathrm{CH}$, Halperin S, Riffelmann $M$, et al. Pertussis of adults and infants. Lancet Infect Dis 2002;2:744-50.

33 Ryan M, Murphy G, Gothefors L, et al. Bordetella pertussis respiratory infection in children is associated with preferential activation of type 1 Thelper cells. J Infect Dis 1997; 175:1246-50.

$34 \mathrm{Kim}$ YS, Kwon KS, Kim DK, et al. Inhibition of murine allergic airway disease by Bordetella pertussis. Immunology 2004;112:624-30.

35 Martin RJ, Kraft M, Chu HW, et al. A link between chronic asthma and chronic infection. J Allergy Clin Immunol 2001;107:595-601.

36 Sigurs N, Bjarnason R, Sigurbergsson F, et al. Respiratory syncytial virus bronchiolitis in infancy is an important risk factor for asthma and allergy at age 7. Am J Respir Crit Care Med 2000;161:1501-7.

37 Talbot TR, Hartert TV, Mitchel E, et al. Asthma as a risk factor for invasive pneumococcal disease. N Engl J Med 2005;352:2082-90. 